\title{
Review of: "Directed acyclic graphs to explore causality in Epidemiological study designs, part I: an introduction to DAGs"
}

\section{Indranath Chatterjee}

1 Tongmyong University

Potential competing interests: The author(s) declared that no potential competing interests exist.

This article is well written in an explanatory manner. I am quite impressed with the explanation of the causality to solve the major issue. 